\title{
Inversion of the 3-D exponential X-ray transform for a half equatorial band and other semi-circular geometries
}

\author{
Frédéric Noo ${ }^{1}$, Rolf Clackdoyle ${ }^{1}$, Jean-Marc Wagner ${ }^{2}$ \\ ${ }^{1}$ Department of Radiology, University of Utah, U.S.A. \\ ${ }^{2}$ Department of Telecommunications, University of Liège, Belgium.
}

\begin{abstract}
This work presents new mathematical results on the inversion of the exponential X-ray transform. It is shown that a reconstruction formula can be obtained for any data set whose projection directions consist of a union of half great circles on the unit sphere. A basic example of such a data set is the semi equatorial band; the discussion in the paper is mostly focussed on this example. The reconstruction formula takes the form of a Neumann (geometric) series and is both exact and stable.

The exponential X-ray transform has been mainly studied in SPECT imaging. In this context, our results demonstrate mathematically that fully 3-D image reconstruction in SPECT with non-zero attenuation does not always require symmetric data sets (opposing views).
\end{abstract}

Short title: inversion of the exponential x-ray transform

Corresponding address:

Frédéric Noo, Ph.D.

University of Utah, Department of Radiology, CAMT Building, 729 Arapeen Drive

Salt Lake city, Utah 84112, U.S.A.

Tel: $+1-801-585-1370$

Fax: +1-801-585-3592

E-mail: noo@doug.med.utah.edu 


\section{Introduction}

The inversion of the exponential X-ray transform is a generalization to three dimensions of the inversion of the exponential Radon transform in two dimensions. The problem is to reconstruct a three-dimensional (3-D) image $f$ from exponentially-weighted parallel-beam projections

$$
p(\underline{\theta}, \underline{s})=\int_{-\infty}^{+\infty} d t f(\underline{s}+t \underline{\theta}) \exp (\mu t)
$$

where $\underline{\theta}$ is the direction of projection, $\mu$ is a constant, and vector $\underline{s}$ is orthogonal to $\underline{\theta}$ and is used to specify different lines of integration in the direction $\underline{\theta}$. Exponential parallel-beam projections can be used to model the data in SPECT imaging when the activity is contained in a convex region of constant attenuation $\mu$ (see Markoe, 1984). In that case, the vector $\underline{\theta}$ is defined by the orientation of the camera and the collimator holes, and $\underline{s}$ is used to specify detector locations for the $p(\underline{\theta},$.$) projection.$

The set of directions $\underline{\theta}$ for which $p$ is measured defines the data acquisition geometry. This set, denoted $\Omega$, is a subset of the unit sphere. The most common set $\Omega$ encountered in SPECT imaging is the great circle $\left(360^{\circ} \mathrm{scan}\right)$ or half great circle $\left(180^{\circ} \mathrm{scan}\right)$ of directions orthogonal to the patient bed. However, fully 3-D geometries are also possible, such as the rotating slant-hole SPECT geometry (Clack et al., 1996).

Image reconstruction from exponential X-ray projections on a great circle has been widely studied over the last twenty years and is now well-understood, particularly due to the significant works of Tretiak and Metz (1980), Metz and Pan (1995) and Pan and Metz (1995). In fully 3-D geometries, the situation is very different. To our knowledge, only four works concerning exact fully 3-D reconstruction from exponential X-ray projections have been published. One of these (Palamodov, 1995) applies to any symmetrical data set $^{1}$ provided the diameter of the region where $f$ is non-zero is below some bound depending on $\mu$. The other three (Hazou 1988, Weng et al. 1996, and Wagner and Noo 2001) impose no restriction on the object diameter but only apply to specific symmetrical geometries, the most general of which is a union of great circles.

Currently, the class of data sets for which an inversion formula of the imaging equation (1) exists is unknown. It is not even known what conditions a data set must satisfy to be complete. The 3-D reconstruction theory for X-ray projections $(\mu=0)$ (Orlov 1975, Defrise et al. 1989) is not easily modified to handle exponential $\mathrm{X}$-ray projections.

In this paper, we show that an exact inversion of the exponential X-ray transform is possible for a large class of fully 3 -D non-symmetrical geometries, called semi-circular geometries. A semi-circular geometry is defined as any set $\Omega$ that is a union of half great circles on the unit sphere. A basic example of such a geometry is the half equatorial band $\Omega_{h e b}$ illustrated in figure 1a; most of the discussion in the paper concentrates on this example. Another example is a circular scan of 270 degrees (a three-quarters great circle).

In principle, the half equatorial band and the three-quarters great circle geometries could be implemented on existing SPECT scanners. However, the practicabilities of such geometries

\footnotetext{
${ }^{1}$ A data set $\Omega$ is symmetrical when $\underline{\theta} \in \Omega$ implies $-\underline{\theta} \in \Omega$.
} 
is not the subject of this paper. These geometries are provided as examples to enhance the readibility of the manuscript and to indicate that the theoretical developments of this paper are not irrelevant to SPECT imaging techniques. The theoretical advances presented in this paper provide another step towards a complete understanding of the exponential and attenuated X-ray transform, which is of key importance for the development of future imaging systems.

The basic idea for reconstruction with semi-circular geometries is to combine a recent result on reconstruction from $180^{\circ}$ scans (Noo and Wagner 2001) with the true three-dimensional reconstruction (TTR) method of Ra et al. (1982). As discussed at the beginning of section 2, the semi equatorial band $\Omega_{h e b}$ can be seen as a union of half great circles. In Noo and Wagner (2001), it was shown in a 2-D context that an integral equation for $f$ can be obtained from data on a half great circle. Using this result, an integral equation for $f$ is written in section 2 for any half great circle in $\Omega_{h e b}$. Next, in section 3 , all integral equations are combined together to obtain a single integral equation for $f$, which involves all data from $\Omega_{h e b}$. This combination step is similar to the TTR method of Ra et al. (1982). In section 4, the integral equation of section 3 is shown to admit a unique and stable solution in form of a Neumann series. A reconstruction algorithm is given at the end of that section. Results obtained from computer-simulated data are presented in section 5. Finally, section 6 provides a discussion to give more insight into sections 2 to 4 and to suggest how the method can be applied to other geometries such as a circular scan of 270 degrees (a three-quarters great circle).

\section{A half great circle in the semi equatorial band}

Consider a full equatorial band on the unit sphere and select the points of this band that lie on the same side of a plane passing through the $z$-axis. The union of these points is a half equatorial band. Figure 1a gives an illustration of a half equatorial band of aperture $2 \theta_{0}$; for this example, the restricting plane is the $(y, z)$-plane and the selected points are on the negative- $x$ side. The mathematical definition of this half equatorial band is $\Omega_{h e b}=$ $\left\{\underline{\theta}=\left(\theta_{x}, \theta_{y}, \theta_{z}\right):|| \underline{\theta}||=1,\left|\theta_{z}\right| \leq \cos \theta_{0}, \theta_{x} \leq 0\right\}$.

It can be shown that $\Omega_{h e b}$ is a union of half great circles. First note that a half great circle is the part of a great circle that lies one side of a plane passing through the center of the unit sphere. Next, consider the full equatorial band $\Omega_{f e b}$ used to define $\Omega_{h e b}$. From Ra et al. (1982), it is known that each point in $\Omega_{f e b}$ lies on at least one great circle that is fully contained in $\Omega_{f e b}$. Therefore, each point in $\Omega_{h e b}$ is on at least one great circle that is fully contained in $\Omega_{f e b}$. The part of this great circle lying on the negative- $x$ side of the $(y, z)$-plane is a half great circle, which furthermore is completely contained in $\Omega_{h e b}$. Hence, each point in $\Omega_{h e b}$ is on a half great circle that is fully contained in $\Omega_{h e b}$, i.e $\Omega_{h e b}$ is a union of half great circles.

In this section, a single half great circle in $\Omega_{h e b}$ is considered (see figures $1 \mathrm{a}$ and $1 \mathrm{~b}$ ). The notation $\mathcal{I}(\underline{n})$ is used to describe one of these half great circles, with $\underline{n}$ defined as the unique unit vector with positive $z$-component and orthogonal to $\mathcal{I}$. Let $E$ be the endpoint of $\mathcal{I}(\underline{n})$ with positive $y$-component, necessarily lying in the $x=0$ plane. Let $\underline{b}=\underline{O E}$ where $O$ is the center of the sphere and let $\underline{a}=\underline{n} \times \underline{b}$. 
Now, consider equation (1) for $\underline{\theta}$ in a half-circle $\mathcal{I}(\underline{n})$. From the results in Noo and Wagner (2001), it is known that $f$ satisfies the following integral equation

$$
f(\underline{x})=f_{0}(\underline{x}, \underline{n})+w(\underline{x}, \underline{n}) * f(\underline{x})
$$

where the symbol $*$ denotes a $3 \mathrm{D}$ convolution and where $f_{0}(\underline{x}, \underline{n})$ and $w(\underline{x}, \underline{n})$ are defined as follows. The function $f_{0}(\underline{x}, \underline{n})$ is the result of (incorrectly) applying the filtered backprojection (FBP) algorithm of Tretiak and Metz (1980) to the projections from the half great circle $\mathcal{I}(\underline{n})$ :

$$
f_{0}(\underline{x}, \underline{n})=\int_{\mathcal{I}(\underline{n})} d \underline{\theta} p_{0}^{F}(\underline{\theta}, \underline{x}-(\underline{x} \cdot \underline{\theta}) \underline{\theta}) \exp (-\mu \underline{x} \cdot \underline{\theta})
$$

with

$$
p_{0}^{F}(\underline{\theta}, \underline{s})=\int_{\underline{s}^{\prime} \cdot \underline{\theta}=0} d \underline{s}^{\prime} p\left(\underline{\theta}, \underline{s}-\underline{s}^{\prime}\right) \delta\left(\underline{s}^{\prime} \cdot \underline{n}\right) k_{\mu}\left(\underline{s}^{\prime} \cdot(\underline{n} \times \underline{\theta})\right)
$$

where $\delta$ is the $1 \mathrm{D}$ dirac function and $k_{\mu}$ is the notch filter

$$
k_{\mu}(r)=1 / 2 \int_{|\nu|>\mu / 2 \pi} d \nu|\nu| \exp (j 2 \pi r \nu) .
$$

The convolution kernel is given by $w(\underline{x}, \underline{n})=\delta(\underline{x} \cdot \underline{n}) \hat{w}(\underline{x} \cdot \underline{a}, \underline{x} \cdot \underline{b})$ where

$$
\begin{aligned}
\hat{w}(u, v)=-\frac{\sinh \mu v}{\pi v} q(u)+\frac{\bar{\mu}}{\pi u}\left\{\frac{2 \sinh \mu v}{\mu v}-\right. & \\
& \left.\frac{\sinh \mu(v+i u)}{\mu(v+i u)}-\frac{\sinh \mu(v-i u)}{\mu(v-i u)}\right\}
\end{aligned}
$$

with

$$
q(u)=-\int_{\mathbb{R}} d \sigma i \operatorname{sign}(\sigma) \exp (i 2 \pi u \sigma)
$$

By definition, $q(u)$ is the convolution kernel of the Hilbert transform. Note that $w(\underline{x}, \underline{n})$ is an odd function in $\underline{x}$, i.e $w(-\underline{x}, \underline{n})=-w(\underline{x}, \underline{n})$.

Basically, for exponential projections $p(\underline{\theta}, \underline{s})$ measured on the $\left(360^{\circ}\right)$ great circle $\mathcal{C}(\underline{n})$ of unit vectors orthogonal to $\underline{n}$, the Tretiak and Metz FBP gives an accurate reconstruction $f$. However, on the $\left(180^{\circ}\right)$ half circle $\mathcal{I}(\underline{n})$, the reconstruction gives an incorrect $f_{0}$. The images $f_{0}$ and $f$ are related according to equation (2), which involves $w$.

\section{The semi equatorial band}

The discussion is now directed at the half equatorial band of figure 1a. Let $A$ be the set of unit vectors corresponding to all the half great circles in $\Omega_{h e b}$. See figure $1 b$.

By averaging together the integral equations $(2)$ available for each half-circle $\mathcal{I}(\underline{n})$ in $\Omega_{h e b}$, an integral equation involving all the data from $\Omega_{h e b}$ is obtained for $f$ :

$$
f(\underline{x})=f_{h e b}(\underline{x})+W(\underline{x}) * f(\underline{x})
$$


with

$$
f_{h e b}(\underline{x})=c \int_{A} d \underline{n} f_{0}(\underline{x}, \underline{n})
$$

and

$$
W(\underline{x})=c \int_{A} d \underline{n} w(\underline{x}, \underline{n}),
$$

where $c$ is a normalization constant;

$$
c=1 / \int_{A} d \underline{n}=1 /\left(2 \pi\left(1-\cos \theta_{0}\right)\right)
$$

if $\theta_{0}$ is the half aperture of the band.

Following the same argument as in Wagner and Noo $(2001)$, it is now shown that $f_{\text {heb }}(\underline{x})$ can be calculated in a fully 3 -D FBP way. As a first step, equation (3) for $f_{0}(\underline{x}, \underline{n})$ is rewritten in the form

$$
f_{0}(\underline{x}, \underline{n})=\int_{\Omega_{h e b}} d \underline{\theta} \delta(\underline{\theta} \cdot \underline{n}) p_{0}^{F}(\underline{\theta}, \underline{x}-(\underline{x} \cdot \underline{\theta}) \underline{\theta}) \exp (-\mu \underline{x} \cdot \underline{\theta}) .
$$

Next, this expression for $f_{0}$ with $p_{0}^{F}$ given by (4) is inserted in (9) and the integration over $\underline{n} \in A$ is moved inside the convolution integral for $p_{0}^{F}$. The result is the FBP formula

$$
f_{h e b}(\underline{x})=\int_{\Omega_{h e b}} d \underline{\theta} p^{F}(\underline{\theta}, \underline{x}-(\underline{x} \cdot \underline{\theta}) \underline{\theta}) \exp (-\mu \underline{x} \cdot \underline{\theta})
$$

where $p^{F}(\underline{\theta}, \underline{s})$ is obtained from $p(\underline{\theta}, \underline{s})$ using the convolution equation

$$
p^{F}(\underline{\theta}, \underline{s})=\int_{\underline{s}^{\prime} \cdot \underline{\theta}=0} d \underline{s}^{\prime} p\left(\underline{\theta}, \underline{s}-\underline{s}^{\prime}\right) h_{\mu}\left(\underline{\theta}, \underline{s}^{\prime}\right)
$$

with

$$
h_{\mu}(\underline{\theta}, \underline{s})=c \int_{A} d \underline{n} \delta(\underline{\theta} \cdot \underline{n}) \delta(\underline{s} \cdot \underline{n}) k_{\mu}(\underline{s} \cdot(\underline{n} \times \underline{\theta})) .
$$

It is observed that $h_{\mu}$ is identical to the filter obtained in Wagner and Noo (2001) for FBP reconstruction from exponential $\mathrm{X}$-ray projections on a full equatorial band.

As readily seen from its definition, the filter $h_{\mu}$ is a generalized function with singularities at $\underline{s}=0$. The implementation of equation (14) therefore requires the use of some regularization technique. For an accurate computation of $p^{F}(\underline{\theta}, \underline{s})$ from samples of $p(\underline{\theta}, \underline{s})$ on a Cartesian grid, (14) should be implemented in the Fourier domain with some apodizing frequency window, such as the Hanning window. Such an implementation requires the knowledge of the Fourier transform of the filter $h_{\mu}$. It is shown in Wagner and Noo (2001) that this transform is

$$
\begin{aligned}
H_{\mu}(\underline{\theta}, \underline{\nu}) & =\int_{\underline{s} \cdot \underline{\theta}=0} d \underline{s} h_{\mu}(\underline{\theta}, \underline{s}) \exp (-j 2 \pi \underline{s} \cdot \underline{\nu}) \\
& =\frac{c}{2} \int_{\mathcal{C}_{\mu}(\underline{\theta}) \cap A} d \underline{n}|\underline{\nu} \cdot(\underline{n} \times \underline{\theta})|, \quad \underline{\nu} \cdot \underline{\theta}=0
\end{aligned}
$$


where $\mathcal{C}_{\mu}(\underline{\theta})$ is a subset of the great circle $\mathcal{C}(\underline{\theta})$ of unit vectors orthogonal to $\underline{\theta}$ :

$$
\mathcal{C}_{\mu}(\underline{\theta})=\mathcal{C}(\underline{\theta}) \backslash\{\underline{n} \in \mathcal{C}(\underline{\theta}):|\underline{\nu} \cdot(\underline{n} \times \underline{\theta})|<\mu / 2 \pi\} .
$$

Note, in particular, that $\mathcal{C}_{\mu}(\underline{\theta})$ is empty when ||$\underline{\nu}||<\mu / 2 \pi$ because $|\underline{\nu} \cdot(\underline{n} \times \underline{\theta})|<\mu / 2 \pi$ for any $\underline{n}$ in this case. Therefore, $H_{\mu}(\underline{\theta}, \underline{\nu})=0$ if $\|\underline{\nu}\|<\mu / 2 \pi$. Table 1 in Wagner and Noo (2001) can be directly used to get the values of integral (16) for $\underline{\theta} \in \Omega_{\text {heb }}$ and $\underline{s}$ such that $\underline{s} \cdot \underline{\theta}=0$.

\section{Solution of the integral equation}

In this section, it is shown that the integral equation (8) admits a unique solution which can be expressed in the form of a Neumann series.

The main problem encountered in the solution of (8) is the exponential growth of the filter $W(\underline{x})$ with increasing $\underline{x}$. This growth is evident in equation (6) used for the definition of $w(\underline{x}, \underline{n})$ which appears in the definition of $W(\underline{x})$ (equation (10)). Because of this behavior at large $\underline{x}$ values, $w(\underline{x})$ does not admit a Fourier transform and (8) cannot be solved by simple deconvolution.

Let $\mathcal{D}$ be the sphere of radius $R$ such that $f(\underline{x})=0$ for $\underline{x} \notin \mathcal{D}$ and let

$$
\chi(\underline{x})= \begin{cases}1 & \text { if } \underline{x} \in \mathcal{D} \\ 0 & \text { otherwise }\end{cases}
$$

In practice $R$ is always finite since $f$ is physically restricted to a finite region. Since $\chi f=f$, equation (8) can be rewritten in the form

$$
f=\chi f_{h e b}+K f
$$

where the operator $K$ is defined by $K f=\chi(W * f)$. Equation (19) can be solved using results from functional analysis. Let $I$ be the identity operator. From (19), it is clear that

$$
(I-K) f=\chi f_{h e b} .
$$

Hence, using the geometric series $(I-K)^{-1}=\sum_{m=0}^{\infty} K^{m}$,

$$
f=\sum_{m=0}^{\infty} K^{m} \chi f_{h e b} .
$$

The convergence of the geometric series and the existence of $(I-K)^{-1}$ are ensured if $\|K\|<1$ where $\|K\|$ denotes the norm of $K$. To define $\|K\|$, the domain of application of equation (19)

must be first defined. From the results in Noo and Wagner (2001), it can be shown that $K$ maps $L^{2}(\mathcal{D})$ onto $L^{2}(\mathcal{D})$ where $L^{2}(\mathcal{D})$ is the space of square integrable functions in $\mathcal{D}$. So, (19) is valid in $L^{2}(\mathcal{D})$ with

$$
\|K\|^{2}=\sup _{f \in L^{2}(\mathcal{D})} \frac{(K f, K f)}{(f, f)}
$$


where the expression (.,.) denotes the inner product between functions in $L^{2}(\mathcal{D})$. In addition, it is noted that $K$ is skew-symmetric. That is

$$
(K f, g)=-(f, K g) \quad \text { for any } f, g \in L^{2}(\mathcal{D}) .
$$

This property is a consequence of the oddness of $W(\underline{x}): W(-\underline{x})=-W(\underline{x})$ because $w(-\underline{x}, \underline{n})=$ $-w(\underline{x}, \underline{n})$ (see equations (6) and (10)). Since (23) holds for any $f, g \in L^{2}(\mathcal{D})$ and $K$ is a linear operator, $\|K\|$ is finite (see the theorem of Hellingter-Toeplitz in Kreyszig 1989).

Conceptually, the presence of $\chi$ in the definition of $K$ limits the size of $\|K\|$. However, the condition $\|K\|<1$ is very restrictive and simulations indicate that it is only met for small values of $R$. To overcome this difficulty, the skew-symmetry of $K$ is invoked. Using the same approach as in Noo and Wagner (2001), a modified operator $\hat{K}=(1-\gamma) I+\gamma K$ is introduced using some parameter $\gamma \in(0,1)$. Equation (19) is equivalent to

$$
f=\gamma \chi f_{h e b}+\hat{K} f .
$$

Next, it is observed from (22) and (23) that $\|\hat{K}\|^{2}=(1-\gamma)^{2}+\gamma^{2}\|K\|^{2}$. The minimum of this expression is at $\gamma=\gamma_{\text {opt }}=1 /\left(1+\|K\|^{2}\right)$. For this value of $\gamma, \hat{K}=\hat{K}_{\text {opt }}$ with

$$
\left\|\hat{K}_{\text {opt }}\right\|=\frac{\|K\|}{\left(1+\|K\|^{2}\right)^{1 / 2}}
$$

which is always smaller than one. Therefore, (24) admits a unique solution

$$
f=\gamma_{o p t} \sum_{m=0}^{\infty} \hat{K}_{o p t}^{m} \chi f_{h e b}
$$

because the geometric series $\left(I-\hat{K}_{\text {opt }}\right)^{-1}=\sum_{m=0}^{\infty} \hat{K}_{o p t}^{m}$ always converges.

The reconstruction of $f$ from formula (26) can be implemented in the following way:

- Step 1: Compute $\chi f_{\text {heb }}$ from the data $p$ on $\Omega_{h e b}$ using (13).

- Step 2: Compute $f_{n}=\hat{K}_{\text {opt }} \chi f_{n-1}$ for $n=1, \ldots, N$.

- Step 3: Compute $f_{N}=\gamma_{o p t} \chi f_{h e b}+\gamma \sum_{n=1}^{N} f_{n}$.

The function $f_{N}$ represents the reconstructed image. The accuracy $\left\|f_{N}-f\right\|$ of the reconstruction depends on $\|K\|$. In the absence of noise, the smaller $\|K\|$, the smaller $\|\hat{K}\|$ and thus the smaller the number of terms $N$ required for a given accuracy because the series converges faster.

\section{A numerical example}

A reconstruction was performed from computer-simulated projection data. The original object was a highly idealized heart model made up of three ellipsoids of constant intensity. The two small ellipsoids, representing the ventricles, had a relative intensity $20 \%$ of that in the myocardial 
region. The top row of figure 2 shows a coronal and a transverse slice through the heart phantom. Simulated exponential parallel-beam projections were calculated analytically for $120 \times$ 30 projections on a semi equatorial band of aperture $\pm 25^{\circ}$. Each projection was made up of $128 \times 128$ square pixels of side $1.2 \mathrm{~mm}$. The attenuation coefficient was $\mu=0.152 / \mathrm{cm}$.

The initial image $f_{h e b}$ was obtained by $3 \mathrm{D}$ FBP using the filter given by equation (16). The second row of figure 2 illustrates two slices through $f_{h e b}$, showing poor recovery of the intensity values although the overall features were recovered well. The operator $\hat{K}$ used for step 2 was obtained as follows. Using the analytic form of $w(\underline{x}, \underline{n})$, a numerical integration was performed to obtain $W(\underline{x})$ according to (10). The norm of the operator $K$ was then computed numerically from which $\gamma_{o p t}$ was obtained. The operation of $\hat{K}$ was performed from the stored convolution kernel $W(\underline{x})$ and using $\gamma_{o p t}$. Ten terms were used in the series and the bottom row of figure 2 shows the succesfull recovery of $f$. Each term took about 5 minutes of CPU time on a SUN ULTRA SPARC $10(450 \mathrm{MHz}, 700 \mathrm{Mb}$ RAM $)$.

\section{Discussion and conclusions}

The development of the inversion formula for the semi-equatorial band is based on two fundamental results. The first one is a result of Noo and Wagner (2001) for half great circles of data: data on a half great circle can be used to obtain an integral equation for $f$ with a convolution kernel which is odd. Because the kernel is odd, the integral equation admits a unique solution in the form of the Neumann (geometric) series. The second result is that the sum of integral equations obtained for individual half-circles generates an integral equation of the same form, with an odd convolution kernel. Therefore, for any data set consisting of a union of half great circles, an integral equation for $f$ can be formed which is solvable as a geometric series.

For a data set $\Omega$ defined as a union of half great circles $\mathcal{I}(\underline{n})$ with $\underline{n}$ ranging over a set $A$, the reconstruction steps are the following. First, the initial term of the series (corresponding to $f_{h e b}$ ) must be calculated. Conceptually, the data on each semi great circle can be processed using the FBP method of Tretiak and Metz (1980) to obtain individual reconstructions summed to give the required initial term. This approach can be difficult to implement in practice due to finite sampling considerations. A better approach is to apply a 3-D FBP formula similar to (13) to the data on $\Omega$ with a filter $h_{\mu}$ defined as in equation (15). The FBP filter can be computed numerically if necessary. The second step concerns the convolution kernel $W$. This kernel is computed from the definition of $A$ using equation (10) and will usually be stored digitally. Once $W$ and the initial term are known, $f$ can be computed following the steps described at the end of section 4. These steps require the evaluation of the norm of $K$ to define $\hat{K}_{\text {opt }}$. This norm can be evaluated analytically using the power method. The application of $\hat{K}$ to each intermediate term $f$ is straightforward. The main step is the convolution with $W$, evaluated at all voxels in some predefined set $\mathcal{D}$ outside which $f$ is known to be zero.

At this point, it should be observed that the integral equation for $f$ does not need to be built using all half great circles in $\Omega$, as achieved in section 3 . If there exists two sets of half great circles whose union gives $\Omega$, then an integral equation for $f$ can be built using any of these 
two sets and two different inversion formulas for $f$ can be obtained. These two procedures will behave differently in the presence of data noise because the convolution kernel $W$ of the integral equation will be different and a different initial term of the Neuman series will be obtained. In short, any description of $\Omega$ as a union of half great circles can be used to obtain an inversion formula for $f$ but the particular description will influence the image accuracy in presence of data noise. At this stage, we have no rule on how to pick an optimal description.

To illustrate the above discussion, suppose that the data set consists of a circular scan of 270 degrees (a three-quarters great cirsle): $\Omega=\{\underline{\theta}=(\cos \phi, \sin \phi), \phi \in[0,3 \pi / 2]\}$. There are several ways to decompose this data set as a union of half great circles. A simple way is to view it as the union of two half great circles, defined with $\phi \in[0, \pi]$ and $\phi \in[\pi / 2,3 \pi / 2]$ respectively. Another way is to use the union of the semi great circles $\mathcal{I}(\eta)=\{\underline{\theta}=(\cos \phi, \sin \phi), \phi \in[\eta, \eta+\pi]\}$ generated by varying $\eta$ continuously between 0 and $\pi / 2$. Depending on the selected decomposition, a different integral equation will result for $f$, with a different convolution kernel and a different initial term. The associated geometric series for $f$ may converge at different rates and also the FBP formulas for the initial terms may respond differently to data noise. Whatever decomposition is chosen, however, the method will always give more weight to the data in the central interval $\phi \in[\pi / 2, \pi]$ which suggests that other reconstruction formulas may exist to produce more uniform noise treatment.

The results presented here greatly expand the body of knowledge on the inversion of the exponential X-ray transform. In particular, this work presents the first fully 3-D results for a large class of non-symmetric geometries. (A parallel submission to this conference issue by Wagner et al. addresses certain geometries consisting of a union of circles on the unit sphere. This is a distinct class of geometries, but also includes non-symmetric cases.)

The exponential X-ray transform has been mainly studied in SPECT imaging but is also useful in the determination of optimal beam configurations in Intensity Modulated Radiation Therapy (Braunstein 2000). In the context of SPECT imaging, our results demonstrate mathematically that fully 3-D image reconstruction in SPECT with non-zero attenuation does not always require symmetric data sets (opposing views). When the emission takes place in a convex region of constant attenuation, the SPECT data can be converted into exponential X-ray projections (Markoe 1984) and the results of this paper can be applied. Further work is required to handle situations where the attenuation is not constant in the emission region.

\section{Acknowledgement}

The work of F. Noo and R. Clackdoyle was supported by the National Institutes of Health, grant number R01 HL55610.

\section{References}

Clack R, Christian P E, Defrise M and Welch A E 1996 Image reconstruction for a novel SPECT system with rotating slant-hole collimators Conf. Rec. 1995 IEEE Med. Imag. Conf. 1948-52, 1996 
Defrise M, Townsend D W and Clack R 1989 Three-dimensional image reconstruction from complete projections Phys. Med. Biol. 34(5) 573-87

Hazou I A 1988 Inversion of the exponential X-ray transform. I: Analysis Math. Methods in the Applied Sciences 10(10) 561-74

Kreyszig E 1989 Introductory Functional Analysis with Applications (New-York: Wiley)

Markoe A 1984 Fourier inversion of the attenuated X-ray transform SIAM J. Math. Anal. 15(4) 718-22

Mennessier C, Noo F, Clack R, Bal G and Desbat L 1999 Attenuation correction in SPECT using consistency conditions for the exponential ray transform Phys. Med. Biol. 44 2483-510

Metz C E and Pan X 1995 A unified analysis of exact methods of inverting the 2D exponential Radon transform, with implications for Noise Control in SPECT IEEE Trans. Med. Imag. 14(4) 643-58

Noo F and Wagner J M 2001 Image reconstruction in 2D SPECT with 180-degree acquisition Inverse Problems 17 1357-71

Orlov S S 1975 Theory of three dimensional reconstruction: 1. Conditions of a complete set of projections Sov. Phys.-Crystallogr. 20 312-14

Palamodov V P 1996 An inversion method for an attenuated X-ray transform Inverse Problems 12 $717-29$

Pan X and Metz C E 1995 Analysis of noise properties of a class of exact methods of inverting the 2D exponential Radon transform IEEE Trans. Med. Imag. 14(4) 659-68

Ra J B, Lim C B, Cho Z H, Hilal S K and Correll J 1982 A true three-dimensional reconstruction algorithm for the spherical positron emission tomograph Phys. Med. Biol. 27 37-50

Tretiak O and Metz C 1980 The exponential Radon transform SIAM J. Appl. Math. 39(2) 341-54

Wagner J M and Noo F 2001 Three-dimensional image reconstruction from exponential parallel-beam projections IEEE Transactions on Nuclear Sciences 48(3) 743-49

Weng Y, Zeng G L and Gullberg G T 1996 Filtered backprojection algorithms for attenuated parallel and cone-beam projections sampled on a sphere in Three-dimensional Image Reconstruction in Radiation and Nuclear Medicine ed. P.Grangeat and J.-L. Amans (Dordrecht: Kluwer) 19-34 

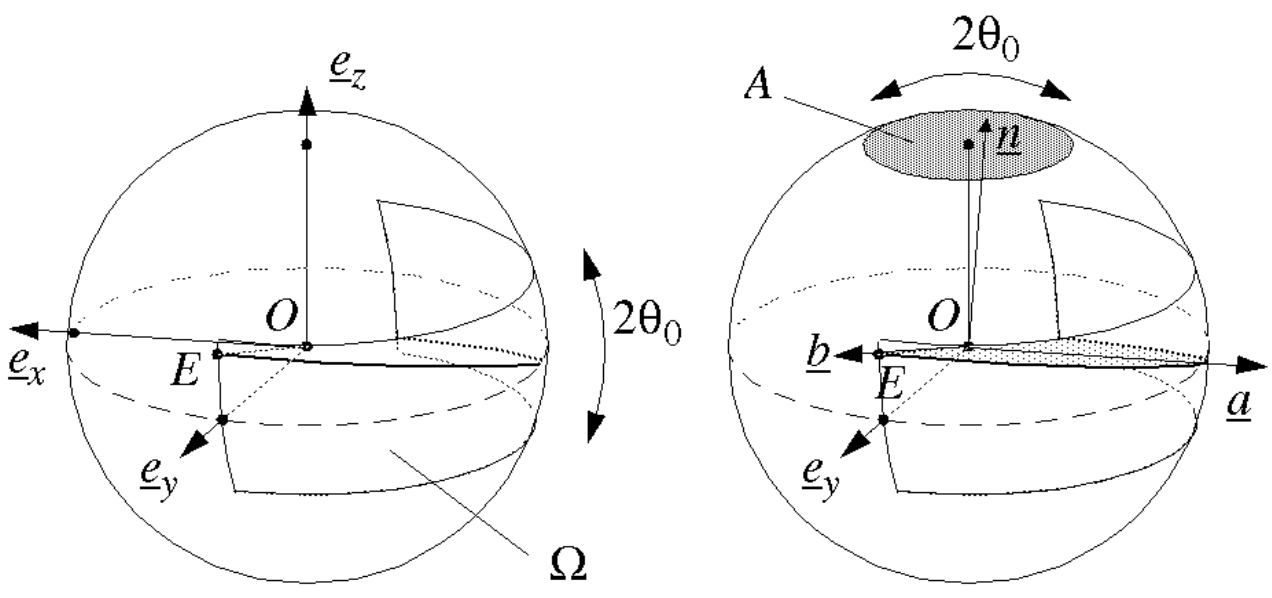

Figure 1: (a-left) Illustration of the half equatorial band including a half great circle. (b-right) Set $A$ of vectors $\underline{n}$ corresponding to all the half great circles in $\Omega$. 

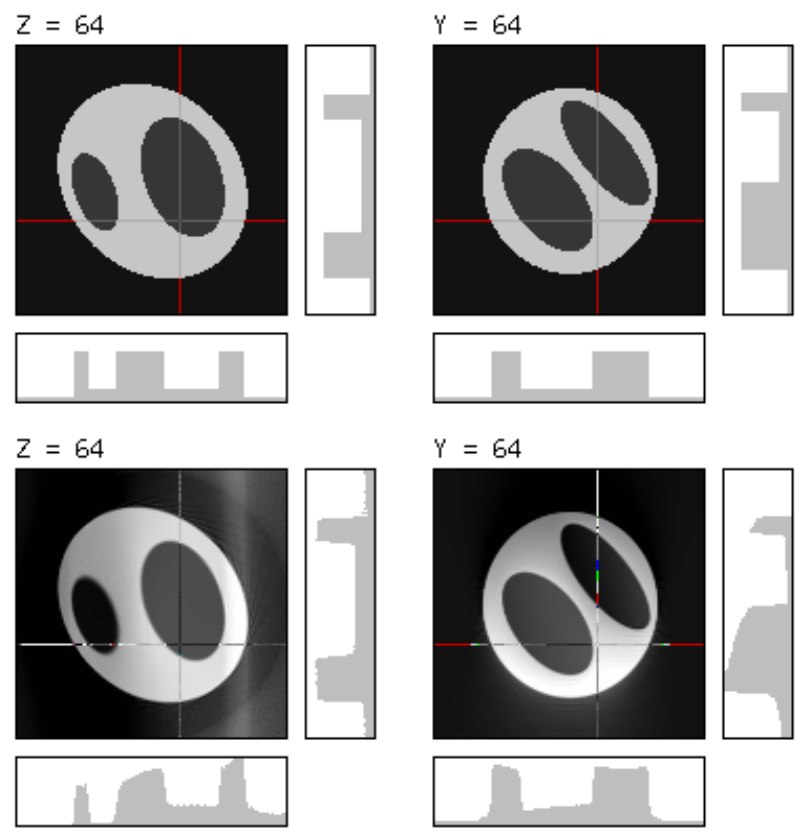

\section{$Y=64$}
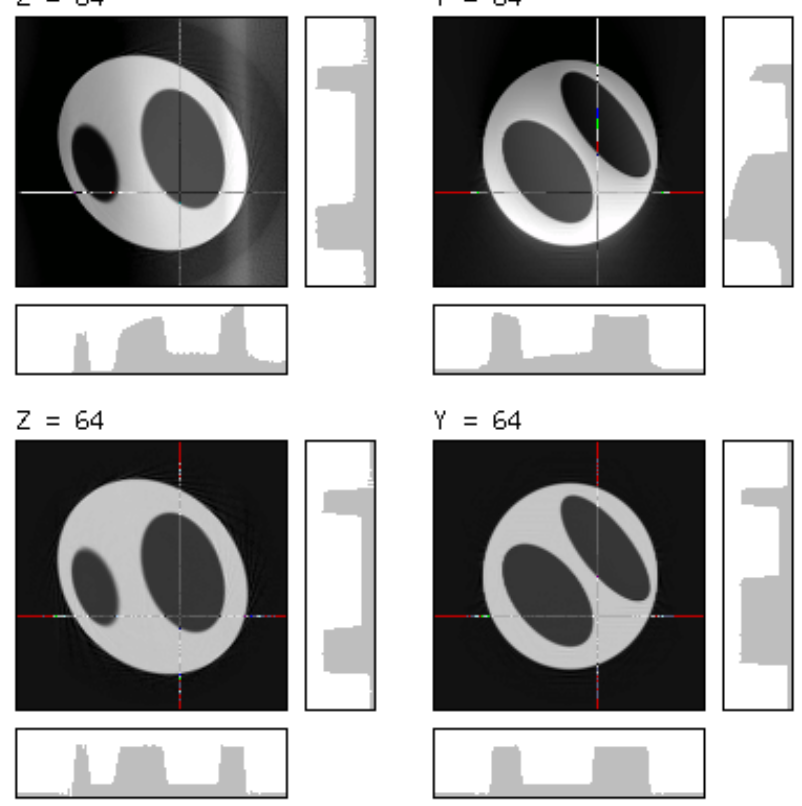

Figure 2: (top) Original phantom, (middle) FBP reconstruction $f_{0}$ using the data on $\Omega$, (bottom) reconstruction using 10 terms of the Neumann series (equation 26). Reconstruction time: about 5 min. cpu per iteration on a SUN ULTRA $10(450 \mathrm{MHz}, 700 \mathrm{Mb}$ RAM). 